\title{
Überarbeitete Richtlinien zur Fest- stellung des Todes treten in Kraft
}

\author{
Jürg Steigera ${ }^{a}$ Michelle Salathéb \\ a Prof. Dr.med., Präsident der Zentralen Ethikkommission (ZEK) der SAMW; ${ }^{\text {b }}$ lic.iur., stv. Generalsekretärin SAMW
}

\begin{abstract}
Das Bundesgesetz über die Transplantation von Organen, Geweben und Zellen (TxG) wurde revidiert und tritt am 15. November 2017 in Kraft. Die Revision des TxG machte eine Überarbeitung der SAMW-Richtlinien «Feststellung des Todes im Hinblick auf Organtransplantationen» erforderlich. Dieser Beitrag fasst die wichtigsten Neuerungen zusammen.
\end{abstract}

Die Voraussetzungen für die Organentnahme sind im Bundesgesetz über die Transplantation von Organen, Geweben und Zellen (TxG) geregelt. In der Frage des Todeskriteriums stützt sich das Gesetz auf die neurologische Definition des Todes, wonach der Mensch tot ist, wenn sämtliche Funktionen seines Hirns, einschliesslich des Hirnstamms, irreversibel ausgefallen sind. Wie der Tod arte legis festgestellt wird, überlässt der Gesetzgeber den medizinischen Experten. Die Verordnung zum TxG verweist auf die Richtlinien «Feststellung des Todes im Hinblick auf Organtransplantationen und Vorbereitung der Organentnahme» der SAMW; die entsprechenden Kapitel haben Rechtskraft.

Voraussichtlich am 15. November 2017 tritt die revidierte Fassung des TxG in Kraft. Die Revision hat unter anderem zwei zentrale Anliegen der SAMW aufgenommen, die zu Unklarheiten in der klinischen Praxis geführt hatten. Diese betreffen:

1. den Zeitpunkt, zu dem mit den Angehörigen über die Organspende gesprochen werden darf;

2. die Frage, welchen vorbereitenden medizinischen Massnahmen die Angehörigen zustimmen können, wenn sich der Patient selbst nicht dazu geäussert hat. Die Revision des TxG machte eine Anpassung der SAMWRichtlinien zur Feststellung des Todes aus dem Jahr 2011 erforderlich. Wie alle medizin-ethischen Richtlinien der SAMW wurden die revidierten Richtlinien von einer interprofessionellen Subkommission ausgearbeitet, hier unter dem Vorsitz von Prof. Jürg Steiger, Basel. Der Entwurf wurde sowohl mit ausgewählten Expertinnen und Experten diskutiert als auch öffentlich zur Vernehmlassung gestellt und anschliessend von SAMWGremien (Zentrale Ethikkommission, Vorstand und Senat) verabschiedet. Die Richtlinien treten per 15. November 2017 in Kraft.
Von den bestehenden Richtlinien aus dem Jahr 2011 wurden die folgenden zentralen Punkte unverändert beibehalten:

- Voraussetzungen für das Todeskriterium (klinische Zeichen);

- Untersuchung durch zwei Fachärzte (Vier-AugenPrinzip), kein Zeitintervall;

- technische Zusatzuntersuchung nur erforderlich, wenn die Voraussetzungen für die klinische Untersuchung nicht erfüllt sind (d. h. wenn die Hirnnerven klinisch nicht untersucht oder potentiell reversible Ursachen als Mitursachen nicht ausgeschlossen werden können);

- keine Organentnahme bei Neugeborenen.

\section{Die zentralen Revisionspunkte im Überblick}

\section{Wann dürfen die Angehörigen zur Organspende} befragt werden?

Der bisherige Gesetzestext liess unklar, ab welchem Zeitpunkt mit den Angehörigen über das Thema der Organspende gesprochen werden darf. Mit den neuen Bestimmungen ist diese Frage nun geklärt. Die klare Trennung zwischen der Entscheidung, eine lebenserhaltende Therapie abzubrechen, und der Entscheidung, Organe zu spenden, ist zentral. Intensivund notfallmedizinische Indikationsstellungen dürfen nicht durch die Option einer Organspende beeinflusst sein. Die Zustimmung zur Organentnahme und zu allfälligen vorbereitenden medizinischen Massnahmen darf deshalb erst eingeholt werden, nachdem der Entscheid feststeht, die lebenserhaltenden Therapien abzubrechen. Gespräche über die Möglichkeit der Organspende dürfen aber bereits vor der Feststellung des 
Todes geführt werden; oft greifen die Angehörigen das Thema selbst auf.

Welche Voraussetzungen müssen erfüllt sein, damit der Prozess zur Feststellung des Todes eingeleitet werden kann?

Bei der Revision der Richtlinien hat die SAMW nicht nur Anpassungen an das revidierte TxG vorgenommen, sondern auch Rückmeldungen aus der Praxis aufgenommen. Als wesentliche Neuerung werden in einem Anhang C zu den Richtlinien die klinischen Voraussetzungen umschrieben, die erfüllt sein müssen, bevor die Feststellung des Todes durchgeführt werden darf. Dies trägt zu mehr Sicherheit im Prozess bei.

\section{Wer darf den Tod feststellen?}

Die überarbeiteten Richtlinien umschreiben die Anforderungen an Ärztinnen und Ärzte, die den Tod feststellen, präziser als bisher. Sie verlangen, dass mindestens einer der beiden beurteilenden Fachärzte zuvor bei mindestens fünf Patienten eine Hirntoddiagnostik unter Supervision durchgeführt hat.

Welche vorbereitenden Massnahmen dürfen bereits vor dem Tod durchgeführt werden und welche Rolle spielen die Angehörigen?

Die Frage, ob und welche vorbereitenden medizinischen Massnahmen vor dem Tod durchgeführt werden dürfen, ist in der Praxis von erheblicher Bedeutung, weil die meisten Patienten auf der Intensivstation und nicht unter voller Therapie versterben. Kommt hinzu, dass nur wenige Patienten eine Spendekarte besitzen und damit die Spendebereitschaft offen bleibt. Um die Organe bis zur Entnahme funktionsfähig zu erhalten, müssen jedoch vorbereitende medizinische Massnahme durchgeführt werden. Mit dem bisherigen TxG blieb unklar, ob Angehörige vorbereitenden Massnahmen vor dem Tod zustimmen können, wenn der betreffende Patient sich selbst nicht dazu geäussert hat. Mit dem revidierten TxG steht nun fest, dass vertretungsberechtigte Angehörige - mit gewissen Ausnahmen - vorbereitenden Massnahmen zustimmen können. Dies gilt auch, wenn sie nicht wissen, wie der Patient entschieden hätte. Ergänzend zum TxG stellen die Richtlinien in einem Flowchart den komplizierten Entscheidungsprozess dar und zählen in einer sog. «Negativliste» diejenigen Massnahmen auf, in deren Durchführung die Angehörigen nicht einwilligen können. Es handelt sich um das Setzen von arteriellen Kanülen und um die Durchführung einer mechanischen Reanimation.

Was gilt, wenn Angehörige aus persönlicher Überzeugung eine Organentnahme ablehnen, obwohl eine Spendekarte vorliegt?

Tatsächlich kommt diese für das Behandlungsteam schwierige Situation im klinischen Alltag vor. Die Richtlinien verweisen auf die rechtliche Regelung, die festhält, dass der Wille der verstorbenen Person demjenigen der nächsten Angehörigen vorgeht. Die Richtlinien empfehlen aber, wenn immer möglich eine einvernehmliche Entscheidung unter allen Beteiligten anzustreben und eine ethische Unterstützung beizuziehen.

Die revidierten Richtlinien unterstützen Betreuungsteams dabei, die geltenden rechtlichen Bestimmungen umzusetzen und mit den vielfältigen ethischen Fragen besser umzugehen. Die Feststellung des Todes beruht zwar auf naturwissenschaftlichen Fakten, bleibt aber auch eine gesellschaftliche Festlegung. Dies zeigt sich unter anderem darin, dass die entsprechenden Verfahrensweisen in verschiedenen Ländern bei aller grundlegenden Gemeinsamkeit in manchen Aspekten divergieren. Das Ziel bleibt, im jeweiligen Kontext für alle Beteiligten die grösstmögliche Gewissheit zu erreichen, dass Spender zum Zeitpunkt der Organentnahme tot sind und der respektvolle Umgang mit dem Sterbenden bzw. dem Leichnam stets gewährleistet ist.

Die Richtlinien stehen mit den genannten Anhängen und Flowcharts auf der SAMW-Website in deutsch, französisch, englisch und italienisch zur Verfügung. Dort können sie auch als gedruckte Broschüre (d/f) bestellt werden: samw.ch/richtlinien 